\title{
ANOMALOUS FADING OF IR-STIMULATED LUMINESCENCE FROM FELDSPAR MINERALS: SOME RESULTS OF THE STUDY
}

\author{
ANATOLY MOLODKOV ${ }^{1}$, IVAR JAEK ${ }^{1,2}$ and VALERY VASILCHENKO ${ }^{2}$ \\ ${ }^{I}$ Research Laboratory for Quaternary Geochronology, \\ Institute of Geology, Tallinn University of Technology, \\ Ehitajate Rd. 5, 19086 Tallinn, Estonia \\ ${ }^{2}$ Institute of Experimental Physics and Technology, \\ University of Tartu, \\ Tähe 4, 51010 Tartu, Estonia
}

Received 2 November 2006

Accepted 4 December 2006

\begin{abstract}
An increasing amount of evidence shows that the use of feldspars in luminescence dating may suffer from significant age underestimates, which are thought to result from anomalous fading. In the hope of finding a solution to the problem we undertook physical investigations of kinetics and temperature dependencies of tunnel afterglow of these minerals. As a result, the method of the direct detection of the tunnel transitions from the dosimetric traps is proposed. Some other relevant results obtained are also presented and discussed. Particularly, the probable reasons of absence of effects of anomalous fading in the feldspar samples investigated in the present work are elucidated.
\end{abstract}

Keywords: IR-OSL dating, feldspars, anomalous fading, ionic processes

\section{INTRODUCTION}

Feldspars are the most abundant and widespread minerals in the Earth's crust. Owing to some favourable palaeodosimetric properties (high luminescence intensity, thermal stability, relatively high saturation dose) this mineral is considered to be one of the most attractive for the luminescence dating of mid-, late Pleistocene and Holocene deposits. However, as it was reported by Wintle (1973) this mineral may suffer from a specific phenomenon known as anomalous fading (AF) whereby luminescence intensity spontaneously declines over time after artificial irradiation, possibly due to localised transition (Templer, 1986) or quantum-mechanical tunnelling (Visocekas, 1985). An increasing number of studies show that most varieties of feldspars fade (Spooner, 1994; Wallinga et al., 2001) and feldspar-based luminescence dates are therefore suspected to be underestimated. This underestimation can sometimes reach up to $30-50 \%$ over the entire dating range of this mineral (Lamothe and Auclair, 1999; Balescu et al., 2003; Huntley and

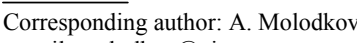

e-mail: molodkov@gi.ee

ISSN 1897-1695 (online) (C) 2007 GADAM Centre,

Institute of Physics, Silesian University of Technology.

All rights reserved.
Lamothe, 2001; Wallinga et al., 2001).

A solution to enable fading feldspars to be used reliably is not yet available, and the fading properties of this mineral are currently still poorly understood. One of the methods frequently used to examine the anomalous fading in the context of geochronology is to carry out a fading test by measuring feldspar samples immediately after artificial irradiation of the feldspars and then after storage at ambient temperature for several weeks or months. Unfortunately, this method is tedious and extremely timeconsuming and often inaccurate due to the scatter of data points and great difference between laboratory and geological time scales. Besides, it may be that the lifetimes of some of the anomalous fading components are too long to be measured on the laboratory time scale. Hence, based on such laboratory fading tests, it is very hard to get an unambiguous indication of the real contribution of the slow fading component into the measured luminescence.

It is clear, therefore, that there is a pressing need for a rapid, reliable method for detection of $\mathrm{AF}$, to allow the estimate of rate, contribution to the post-irradiational luminescence and subsequent correction of the calculated age for $\mathrm{AF}$. 

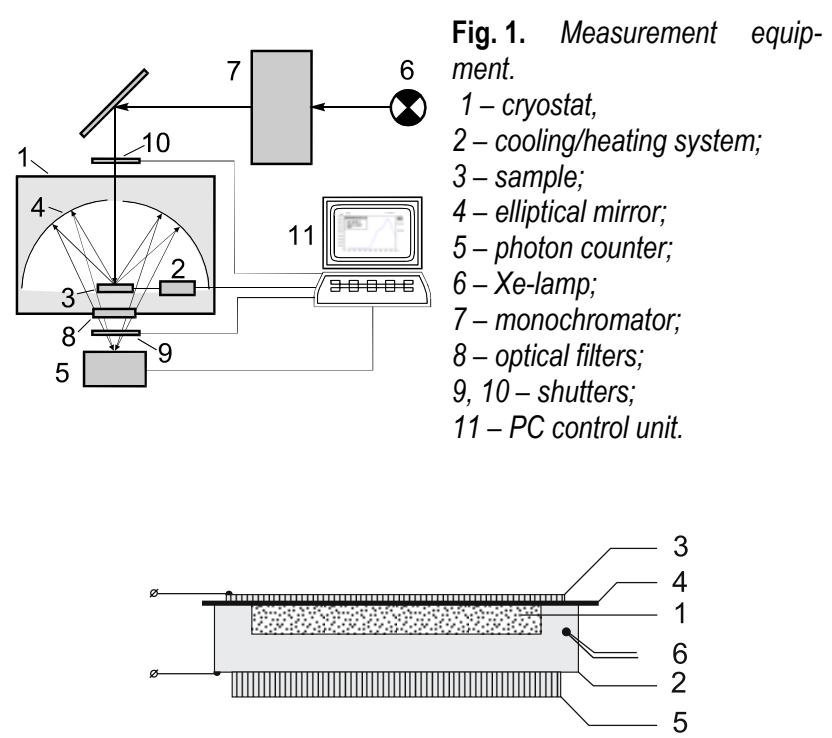

Fig. 2. Ionic conductivity measuring cell. 1 - feldspar; 2 - cell (lower electrode); 3 - upper electrode; 4 - mica; 5 - heater; 6 - thermocouple.

The aim of the present paper is to report our new experimental data on kinetics and temperature dependencies of tunnel afterglow, and tunnel component separation in the optically stimulated luminescence of the feldspars, which is aimed to circumvent the problem of anomalous fading of IR-stimulated luminescence from feldspar minerals.

\section{THE RESEARCH OBJECTS}

Feldspars extracted from numerous samples taken from different areas of Northern Eurasia - from Baltic shores to East-Siberian Sea coast - served as the research objects.

\section{EQUIPMENT}

The equipment consists of two parts, one of which is meant for various optical measurements and the other for electrical measurements.

The equipment of optical measurements (Fig. 1) consists of a cryostat (1) with the cooling/heating system (2) of the sample (3) allowing to carry out optical measurements in the range of LNT up to $450^{\circ} \mathrm{C}$. The cryostat is supplied with an elliptical mirror (4), where the study sample is in one focus and the photon counter (5) (Hamamatsu H6240) in the other. The TL, OSL, optically stimulated afterglow (OSA) and stimulation spectra are measured. At that, TL and temperature dependence of OSL can be measured simultaneously. A Xe-lamp (6) served as a source of stimulating radiation. The necessary wavelength of stimulation is selected by monochromator (7), and the emission band - by optical filters (8). The driving of the shutters $(9,10)$, the control of the experiment and the primary processing of the results of experiment are fulfilled through a PC control unit (11).

Ionic conductivity was measured using a dissipation factor meter BM271 (Fig. 2). This device measures the resistance and capacity of a sample in a parallel equivalent scheme. The operating frequency range of the device is between $100 \mathrm{kHz}$ and $10 \mathrm{MHz}$. The study sample (1) was sprinkled into a groove, $2 \mathrm{~mm}$ in depth and $0.79 \mathrm{~cm}^{2}$ in an area, on a copper plate, which served also as an electrode (2). Another electrode (3) was pushed from above onto the powder sample, which was situated in the measuring cell. A ca $20-\mu \mathrm{m}$-thick mica plate (4) between the second electrode and the studied sample to eliminate the direct galvanic connection between the electrodes and the study sample. The device allows heating of the measuring cell up to $300^{\circ} \mathrm{C}$ and measurement of the temperature dependence of conductivity. Prior to measurement, each sample was dried for a few minutes at $100^{\circ} \mathrm{C}$.

\section{THERMAL AND ATHERMAL BEHAVIOUR OF THE RED AND VIOLET LUMINESCENCE FROM FELDSPARS}

With the purpose to develop an experimental method for correction of dating results we carried out investigations of tunnelling processes in the various feldspars.

Most effectively and easily the tunnel processes can be separated by cooling of irradiated samples, using the effective independence of these processes from temperature, while recombination processes occurring through the conductivity and valence bands "are frozen". In this way Visocekas et al. (1994) and Spooner (1994) came to the conclusion that there is a correlation between the intensity of tunnel processes and anomalous fading, on the one hand, and between the intensity of these processes and the degree of orderliness of the feldspar crystal lattice, on the other.

\section{The temperature dependencies of the red afterglow}

In Fig. 3, the temperature dependencies of the red afterglow of the samples RLQG 1457 and TLN 970 are shown. From the figure it seems that this afterglow contains at least two components, the intensity ratio of which differs in different samples. The intensity of the first component strongly depends on the temperature, reaching a maximum value near the temperature of liquid nitrogen. The second component is almost independent of temperature. It may be assumed that this is the component that becomes apparent in TL at higher temperatures.

Based on the data in Fig. 3, it can be concluded that the first component is subjected to temperature quenching, and the quenching activation energy can be defined; it is about $0.1 \mathrm{eV}$. It also appears that the heating of the investigated samples at $800^{\circ} \mathrm{C}$ and higher temperatures considerably increases the intensity of the tunnel luminescence. We interpret such a phenomenon as a result of the reduction of degree of orderliness of the crystal lattice, caused by heating.

Further we have investigated in detail the time dependence of tunnel afterglow both for the first and second component of this luminescence.

\section{The time dependence of the tunnel afterglow fading}

The time dependence of tunnel afterglow fading of sample RLQG 1457 is given in Fig. 4 in double loga- 


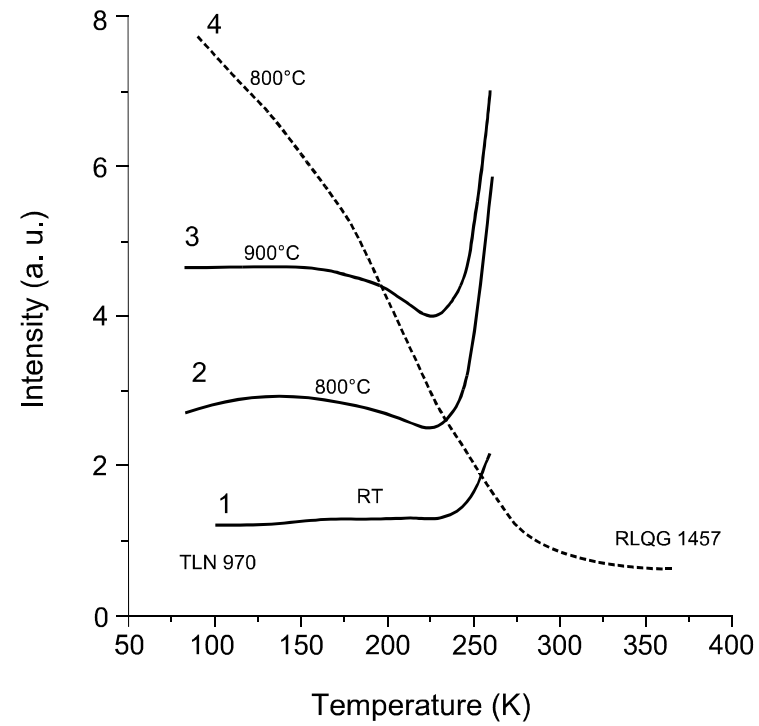

Fig. 3. Temperature dependence of intensity of the red afterglow (longwave-pass filter with a cut-off at $710 \mathrm{~nm}$ ) for samples TLN 970 (curves 1-3) and RLQG 1457 (curve 4). 1 - unheated; 2 - heated at $800^{\circ} \mathrm{C}$ for $15 \mathrm{~min} ; 3$ - heated at $900^{\circ} \mathrm{C}$ for $15 \mathrm{~min}$. X-ray excitation at room temperature (RT); 4 - heated at $800^{\circ} \mathrm{C}$ for $15 \mathrm{~min}$. X-ray excitation at $R T$ followed by heating up to $100^{\circ} \mathrm{C}$

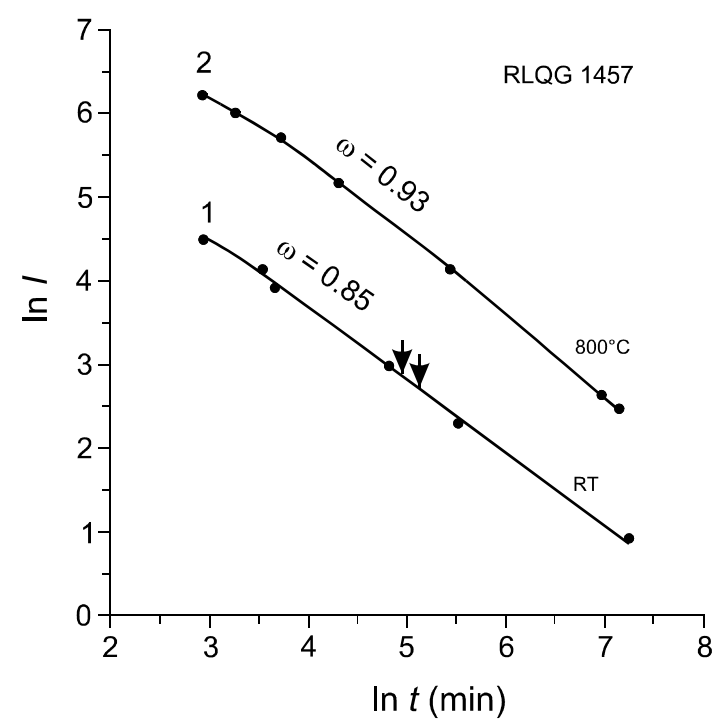

Fig. 4. Time dependence of the red luminescence intensity attenuation at the liquid nitrogen temperature plotted in double logarithmic coordinates. 1 - unheated sample; 2 - heated up to $800^{\circ} \mathrm{C}$; Figures at the curves are values of inclination $\omega$. In the time interval indicated by two arrows the sample has been exposed (at RT) to IR light (900 nm); as a result the signal intensity has decreased in 5 times. $X$-ray excitation at $R T$.

rithmic co-ordinates. It turns out that the fading is well described by the equation:

$I=I_{0} /(1+\kappa t)^{\omega}$

where $I$ is the intensity of the tunnel luminescence at the time $t ; I_{0}$ is the initial intensity at the time $t=0 ; \kappa$ is the parameter characterising the tunnelling probability; $\omega$ is the parameter characterising the order of kinetics.
This means that the fading of the tunnel luminescence in the feldspars follows Becquerel's empirical law. This finding coincides well with that established in the 1970s by Latvian physicists on alkali halogen crystals (Aboltin et al., 1978).

Under condition of $\kappa t » 1$ this law is described in logarithmic co-ordinates by a straight line. Condition $\kappa t » 1$ can be presented as $t \geq t_{0}$ where $t_{0}$ makes up some tens of minutes in the case of feldspars.

The inclination of the straight lines in Fig. 4 will give us the value of $\omega$. For feldspars this value is close to unity, but, as a rule, it is a little lower. From the dosedependence of the attenuation curves (ibid.) some conclusions can be drawn about the detailed mechanism of the tunnel luminescence. Since in our case $\omega$ depends on the dose, and $I_{0}$ is not the linear function of the dose, in general we are dealing not with the tunnelling of the isolated pairs, but with the complex interaction of a number of donor-acceptor pairs.

The data in Fig. 4 also show the influence of the variation of the crystal lattice ordering - in the sample heated at $800^{\circ} \mathrm{C}$ the attenuation curve is situated at a higher level.

However, the most significant result of the research on the tunnel afterglow attenuation is the following: if in the course of the afterglow fading the sample is exposed to the $\lambda=900 \mathrm{~nm}$ infra-red light (i.e. in the band of the dosimetric trap stimulation), so that the number of the charges in these traps considerably decreases (5 times in the given experiment), the attenuation curve does not react to it. From this follows that the red tunnel afterglow is not connected directly to possible tunnelling from the dosimetric traps. A priori such ties are not necessary.

\section{Direct detection of the tunnel process originating from the dosimetric traps}

To detect tunnelling from dosimetric traps it seems necessary to separate tunnel processes in optically stimulated luminescence. This conclusion is based on the results obtained by Kink and Jaek (1972) on alkali halogen crystals as modelling objects; they deduced that, during optical stimulation, traps with double-staged (photothermal) ionisation are involved. The same is the case with the dosimetric traps in feldspars (see Hütt and Jaek, 1989; Duller, 1997) and, therefore, the application of similar methods is justified.

A schematic energy diagram for tunnelling processes during optical stimulation (A) and expected temperature dependence of IR-OSL in Arrhenius co-ordinates $\ln I_{\mathrm{tn}} / I_{0}$ vs $1 / T$ is presented in Fig. 5. This plot represents a straight line. The slope of the line gives the activation energy value $\Delta E$ for thermal ionisation of the trap, excited by the infrared radiation. The inflection point of this line corresponds to the temperature of which the probability of thermal ionisation of the excited trap becomes less than probability of tunnelling; this, to a first approximation, does not depend on temperature. When separating the tunnel processes by such a method, we employ the fact, that in the excited state the effective radius of the electronic cloud of the trap is much more than in the ground state. Figuratively speaking, it is tantamount to a significant reduction of the width of the 
A
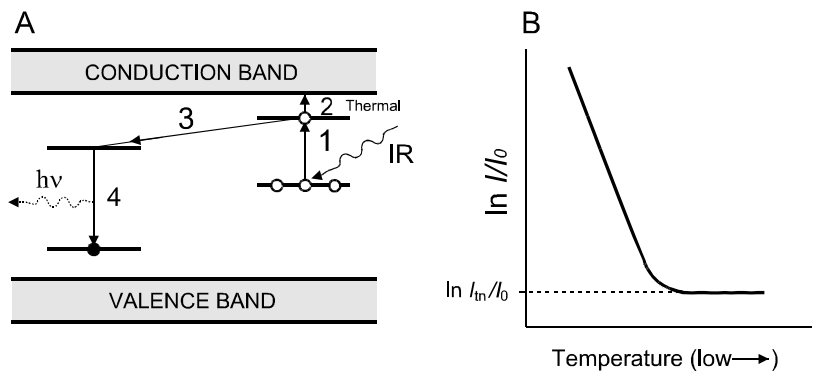

Fig. 5. A) Simplified energy-level diagram explaining tunnelling at photostimulation (after Kink and Jaek, 1972):

1 - photoexcitation of the traps;

2 - thermal ionisation of the excited traps;

3 - tunnel recombination of trapped electron from the excited trap by the ionised luminescence trap;

4 - radiative transition of the excited trap into the basic state;

B) Expected course of the IR-OSL intensity at the lowering of the temperature; I is the intensity at the temperature $T, 10$ is the initial intensity at RT. The dotted line shows in $1 / 10$ value in the temperatureindependent section of the curve with plateau value at $\ln I_{\text {tn }} / l_{0}$, where $I_{\text {tn }}$ is the corresponding IR-OSL intensity. This value characterises the probability of tunnelling process from the excited state of the centre.

potential barrier through which the tunnelling occurs. Then the slow tunnelling causing the fading becomes directly observable at the moment of the photostimulation. When analysing the results it is only necessary to take into account by how many times we have increased the probability of the tunnelling. The value of $\ln I / I_{0}$ in the inflection point of the line on Fig. 5 can be interpreted as a quantitative measure of the tunnelling probability.

Thus, it is necessary to investigate the lowtemperature IR-OSL of feldspars. There is a relatively small number of such works (see, e.g. Bailiff and Barnett, 1994; Rieser et al., 1997).

\section{The low-temperature IR-OSL of feldspars}

In the course of the present investigations the dependencies of $\ln I_{\mathrm{tn}} / I_{0}$ vs $1 / T$ for about 50 different feldspar samples extracted from various deposits, and for some laboratory-doped samples were measured.

Fig. 6 presents typical data on the temperature dependency of feldspar IR-OSL. The reality appears to be richer and more complicated than the idealised model shown in Fig. 5.

Curve 1 in the figure represents the temperature dependence of IR-OSL as it is in the "classical" case. Instead of one straight line with one inflection we see two IR-OSL components with the activation energies $\Delta E_{1}=0.2 \mathrm{eV}$ and $\Delta E_{2}=0.07 \mathrm{eV}$ and, in addition, a lowtemperature section of the curve with $\Delta E_{3} \approx 0 \mathrm{eV}$ not dependent on temperature. This curve is described mathematically by the following equation:

$I(T)=I_{01} e^{-\frac{0.2}{k T}}+I_{02} e^{-\frac{0.07}{k T}}+I_{03}$

The parameters of this formula $I_{01}, I_{02}$ and $I_{03}$ can be easily determined by fitting equation 4.2 to the experimental data. Because the curve has a section with $\Delta E_{3}=0$ (and $I_{03} \neq 0$ ), it is possible to assert that we have recorded the presence of tunnelling in the IR-OSL of feldspar. To
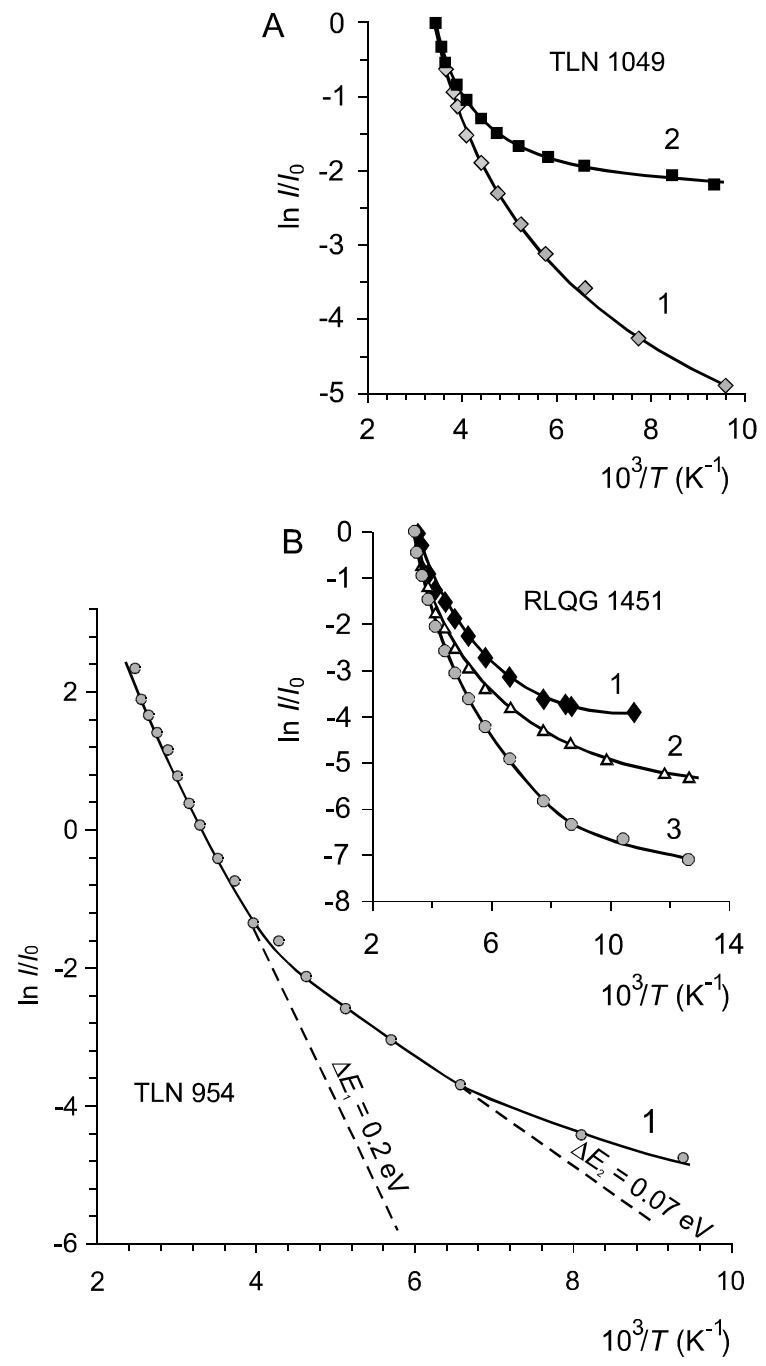

Fig. 6. Temperature dependence of the luminescence intensity in the violet band of the luminescence (370-450 nm filter) of the sample TLN 954. X-ray excitation at $R T$.

Insert A) A plot of In I//o vs 1/T for the sample TLN 1049; 1 - unheated, 2 - heated up to $800^{\circ} \mathrm{C}$.

Insert B) A plot of In I/lo vs $1 / T$ for the sample RLQG 1451. X-ray excitation at RT. 1 - immediately after X-raying; 2 - after $20 \mathrm{~h}$ storing at RT; 3 - after heating at $230^{\circ} \mathrm{C}$ for $5 \mathrm{~min}$.

separate tunnelling from the dosimetric traps (these are the traps with $\Delta E_{1}=0.2 \mathrm{eV}$ ), it is necessary to establish the nature of the component with $\Delta E_{2}=0.07 \mathrm{eV}$.

\section{The nature of the traps with $\Delta \mathrm{E}_{2}=0.07 \mathrm{eV}$}

In the insert A of Fig. 6, the IR-OSL temperature dependencies are shown for a non-heated feldspar sample (1) and for the same sample but preheated up to $800^{\circ} \mathrm{C}$ (2). It can be seen that the tunnelling probability increases following heating. In the insert B, the IR-OSL temperature dependencies for the sample RLQG 1451 are presented for just-irradiated sample (1), after $20 \mathrm{~h}$ storing of the sample at room temperature (2), and after postirradiation annealing at $230^{\circ} \mathrm{C}$ for $5 \mathrm{~min}$ (3). It can be seen that the traps characterised by $\Delta E_{2}=0.07 \mathrm{eV}$, are thermally less stable. Furthermore, $I_{03}$ in equation (4.2) consists of at least two components: 


$$
I_{03}=I_{03}^{\prime}+I_{03}^{\prime \prime}
$$

Of these, $I_{03}^{\prime}$ is connected with tunnelling from the traps having $\Delta E_{2}=0.07 \mathrm{eV}$. It should also be noted that the spectrum of the OSL stimulation under temperature exposures described above practically does not change. Thus it seems that all the traps are of the same type as those with $\Delta E_{1}=0.2 \mathrm{eV}$, but that some have a lower energy of thermal ionisation in both the ground and excited states. The change in the thermal ionisation energy is probably connected with differences in the crystal structure in the local environment of these traps. This suggestion is supported by the increase of the relative number of such traps at the heating above $800^{\circ} \mathrm{C}$.

We pay attention that in the overwhelming majority of cases the dependence $\ln I_{\mathrm{tn}} / I_{0} v s \quad 1 / T$ has monotonous character. This is fair also for the case when the orange band of the luminescence is separated. Some exceptions from this regularity will be considered below.

Coming back to the analysis of the data in Fig. 6, we pay attention to the following point. Relaxation processes in just-irradiated feldspar samples both after storage at room temperature and after thermal treatment, result in a decrease of tunnelling probability, which reaches the minimal value after heating at $230^{\circ} \mathrm{C}$. However, such thermal treatment may have a strong influence on the luminescence properties of feldspars. The preliminary analysis shows that here we are dealing with thermally induced ionic processes.

It is also worth noting that the tunnelling probability in all samples treated at $800^{\circ} \mathrm{C}$ is not less than 5 times higher than in the samples not treated at $800^{\circ} \mathrm{C}$. These results were observed in all 15 samples investigated in this respect. Taking into account data by Mejdahl (1983) about luminescent dating results on the ancient ceramics, showing good agreement with the archaeological data, it is possible to conclude, that the level of tunnelling which is observed after high-temperature treatment of the feldspars is not sufficient to display fading of the tunnel nature in the samples of nonvolcanic origin. Otherwise, an underestimation of the age of the ceramics would be observed.

The reasons mentioned above force us to search for alternative mechanisms for explanation of the fading in the feldspars observed by many researchers. One of the possible alternatives follows from the further analysis of the data in Fig. 6. The high level of tunnel processes with participation of dosimetric levels, observed directly after laboratory irradiation, allows to assume that at least part of IR-OSL depletion at these levels is connected with the tunnel processes in the early stages of relaxation (for TL - completely, see Wintle, 1977). However, characteristic time of attenuation for these processes makes up no more than several days (Fig. 7). After the expiration of this time the tunnelling probability apparently falls well below the level which causes appreciable underestimation of ages.

The other part of the fading of the IR-OSL signal from the dosimetric levels is most likely associated with the relaxation processes in the system of hole levels of capture, including fading associated with the redistribu- tions in system of the centres of different type of radiative and nonradiative recombination. Such redistributions are equivalent to processes of external quenching of luminescence. The fading caused by these processes is illusory, because is not related to change of population of the dosimetric levels. Besides, one has to consider seriously the share of ionic processes in the formation of fading due to preheating. It should be pointed out that the effect of preheating, which refers to ionic processes in feldspars, manifests itself in the following: changes in sensitivity with respect to laboratory irradiation, changes in the ratio of violet and orange bands intensity, shift of radiationinduced TL-peaks compared to unheated samples, but also changes in the thermal activation energy of dosimetric centres excited with IR radiation.

It is quite probable that also in the case when unstable traps are completely emptied by preheating (e.g. at $230^{\circ} \mathrm{C}$ for 5 minutes), then fading of the IR-OSL signal would be expected, but in this case the fading would be thermal (hole or/and ionic process) rather than anomalous.

Finally, we will deal with the problem relating to the applicability of any variety of the red luminescence for dating, which, according to the data by Zink and Visocekas (1997) (TL) and Zhong-Ping Lai et al. (2003) (OSL) does not fade.

\section{The IR-OSL temperature dependencies in different wavebands}

Fig. 8 presents data on the IR-OSL temperature dependencies in the violet and orange bands for sample RLQG 1495. The data presented in the figure do not indicate tunnelling from the dosimetric levels for the orange band. For the violet band the nonmonotonic dependence of luminescence intensity against temperature is observed.

It is interesting to note that a similar nonmonotonic dependence $\ln I / I_{0}$ vs $1 / T$ was also observed by Rieser et al. (1997) in some feldspar samples, but only for the

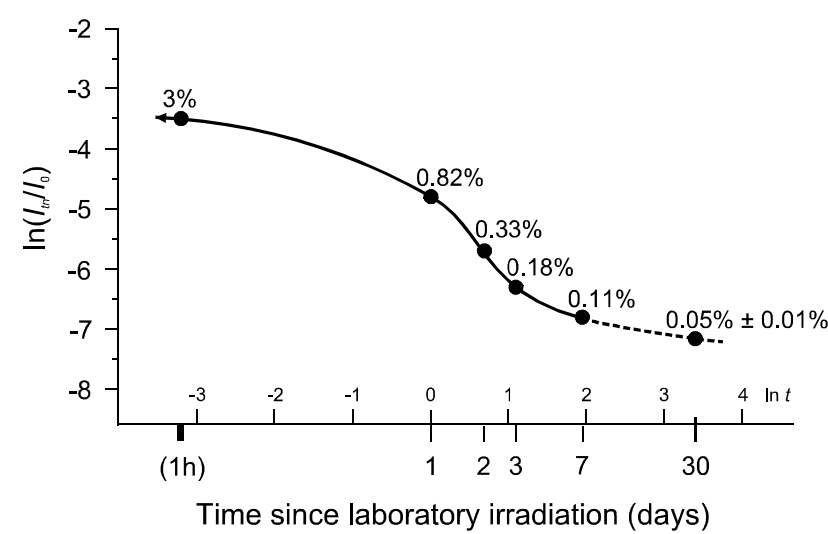

Fig. 7. Tunnelling probability as a function of time since laboratory irradiation for typical feldspar sample from the Scandinavian source region; here $\mathrm{I}_{0}$ is the initial intensity at $R T, \mathrm{I}_{\mathrm{tn}}$ is the plateau value in the temperature-independent section of the course of the IR-OSL intensity recorded with decrease in temperature down to LNT. Figures at the curves are the contributions of the tunnel luminescence to the OSL signal, in percents. The last point was obtained by extrapolation of the curve to the time $t=30$ days. Over 1 month the tunnel luminescence comprises ca $0.05 \%$ of the OSL signal value. 


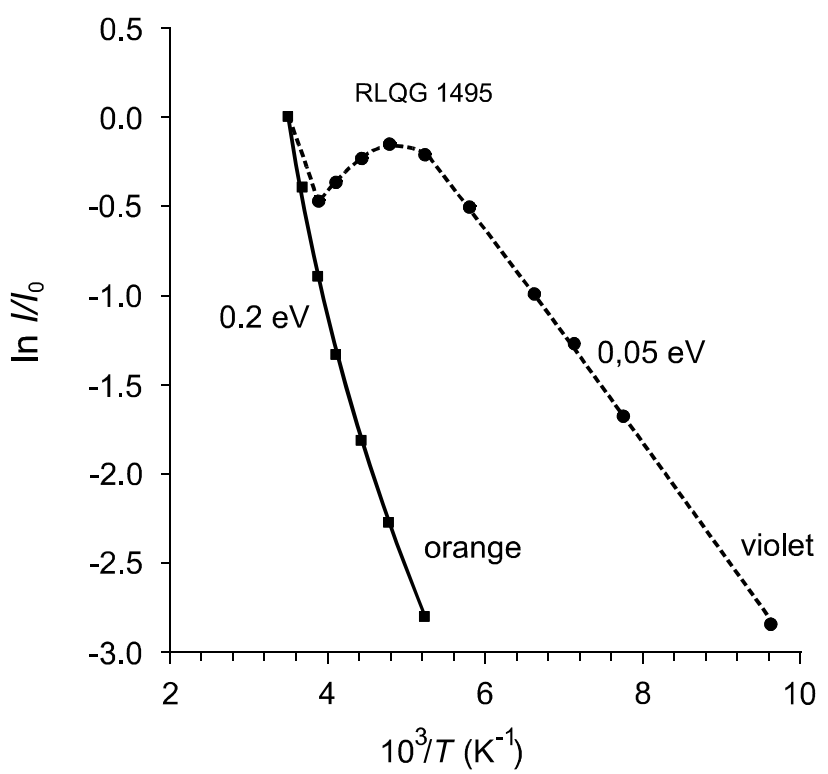

Fig. 8. A plot of In $1 / l_{0}$ vs $1 / \mathrm{T}$ for the sample RLQG 1495 in the violet and orange luminescence bands. $X$-ray excitation at $R T ; \lambda=900 \mathrm{~nm}$.

orange luminescence band. In the most cases we have observed identical (monotonic) behaviour of these dependencies for both bands of the luminescence.

To summarise this section, we note that such a situation when for the one band of the luminescence the tunnelling indications are present, and for another band not (Zink and Visocekas, 1997), while electrons are scooped from the one and the same source (dosimetric levels), is most likely connected with motley phase structure of the samples, extracted from the sediments. In one phase the fading, independent of nature, is present, while in another phase of the same mixture it is absent.

\section{CONCLUSIONS}

In this paper the physical investigations of the palaeodosimetric and optical properties of feldspars from different sites were carried out in hope of providing direct evidence for anomalous fading phenomena. The results of these studies are as follows:

- It is established that the red tunnel afterglow contains, at least, two spectrally close components substantially differing in thermal, kinetic and other properties.

- It is shown that the red tunnel afterglow has no direct connection with the possible tunnel emptying of the dosimetric traps. It would hold for its both components.

- The method of the direct detection of the tunnel transitions from the dosimetric traps is proposed utilising the specific features of the low-temperature (down to LNT) dependencies of the OSL intensity stimulated in the IR region. By this method the indications of tunnelling were revealed for the lattice defects of the same type as the dosimetric traps, which have, however, lower temperature stability. Indications of tunnelling for the stable dosimetric traps within the limits of sensitivity of our light receivers are not revealed neither for the violet, nor for orange luminescence band. It implies a small contribution of the tunnel processes to the fading.

- The intensity of the red tunnel afterglow, as well as the portion of the unstable traps mentioned above, considerably (up to 10 times) increases in the samples heated above $800^{\circ} \mathrm{C}$ in comparison with the same unheated samples. It is obviously connected with the phase transition of the ordered feldspars varieties to the phases with a less ordered crystal lattice. Heating at lower temperatures $\left(100-300^{\circ} \mathrm{C}\right)$ causes also changes in many luminescent properties, which can be interpreted as the result of thermoinduced ionic processes. It can be assumed that some decrease of the dosimetric TL/IR-OSL signal observable in the feldspar samples after laboratory irradiation can really be caused by tunnelling, however, the probability of this process quickly goes down, already within some days after the irradiation.

- The absence of effects of anomalous fading, which is revealed in the coincidence of dating obtained on our study objects with different methods (see, e.g., Molodkov and Bitinas, 2006), is evidently due to the peculiarity of our dating technique - keeping the sample for a month at room temperature instead of its routine preheating which according to the aforesaid, causes changes in luminescent properties in connection with ionic processes.

\section{ACKNOWLEDGEMENTS}

We gratefully acknowledge Tatyana Balakhnichova and Marina Osipova for their contribution to the laboratory work reported here, and Helle Kukk for improving the English text. This research was supported by the grant no. 6112 from the Estonian Science Foundation and by Estonian State Target Funding Project no. 0320080s07.

\section{REFERENCES}

Aboltin DE, Grabovskis VJ, Kangro AR, Luschik ChB, O'KonnelBronel AA, Vitol IK and Zirap VE, 1978. Thermally stimulated and tunnelling luminescence and Frenkel defect recombination in $\mathrm{KCl}$ and $\mathrm{KBr}$ at 4.2 to $77 \mathrm{~K}$. Physica Status Solidi (a) 47: 667-675.

Bailiff IK and Barnett SM, 1994. Characteristics of infrared stimulated luminescence from feldspars at low temperatures. Radiation Measurements 23: 541-546.

Balescu S, Lamothe M, Mercier N, Huot S, Balteanu D, Billard A and Hus J, 2003. Luminescence chronology of Pleistocene loess deposits from Romania: testing methods of age correction for anomalous fading in alkali feldspars. Quaternary Science Reviews 22: 967973.

Duller GAT, 1997. Behavioural studies of stimulated luminescence from feldspars. Radiation Measurements 27: 663-694.

Huntley DJ and Lamothe M, 2001. Ubiquity of anomalous fading in Kfeldspars and the measurement and correction for it in optical dating. Canadian Journal of Earth Sciences 38: 1093-1106.

Hütt $\mathrm{G}$ and Jaek I, 1989. Infrared stimulated photoluminescence dating of sediments. Ancient TL 7: 48-51.

Kink $\mathrm{M}$ and Jaek I, 1972. The tunnelling from exited states of $\mathrm{Tl}^{0}$ centres in $\mathrm{KCl}$ crystals. Transactions of the Institute of Physics and Astronomy of the Academy of Sciences of the ESSR 39: 316-318 (in Russian, with English summary).

Lamothe M and Auclair M, 1999. A solution to anomalous fading and age shortfalls in optical dating feldspar minerals. Earth and Planetary Science Letters 171: 319-323.

Mejdahl V, 1983. Feldspar inclusion dating of ceramics and burnt stones. PACT 9: 351-364. 
Molodkov A and Bitinas A, 2006. Sedimentary record and luminescence chronology of the Lateglacial and Holocene aeolian sediments in Lithuania. Boreas 35: 244-254.

Rieser U, Hütt G, Krbetschek MR and Stoltz W, 1997. Feldspars IRSL emission spectra at high and low temperatures. Radiation Measurements 27: 273-278.

Spooner NA, 1994. The anomalous fading of infra-red stimulated luminescence from feldspars. Radiation Measurements 23: 625632.

Templer RH, 1986. The localised transition model of anomalous fading. Radiation Protection Dosimetry 17: 493-497.

Visocekas R, 1985. Tunnelling radiative recombination in labradorite; its association with anomalous fading of thermoluminescence. $\mathrm{Nu}$ clear Tracks and Radiation Measurements 10: 521-526.

Visocekas R, Spooner NA, Zink A and Blanc P, 1994. Tunnel after glow, fading and infrared emission in thermoluminescence of feldspars. Radiation Measurements 23: 377-385.

Wallinga J, Murray AS, Duller GAT and Törnqvist TE, 2001. Testing optically stimulated luminescence dating of sand-sized quartz and feldspar from fluvial deposits. Earth and Planetary Science Letters 193: 617-630.

Wintle AG, 1973. Anomalous fading of thermoluminescence in mineral samples. Nature 245: 143-144.

Wintle AG, 1977. Detailed study of a mineral exhibiting anomalous fading. Journal of Luminescence 15: 385-393.

Zhong-Ping Lai, Stokes S, Bailey R, Fattahi M and Arnold L, 2003. Infrared stimulated red luminescence from Chinese loess: basic observations. Quaternary Science Reviews 22: 961-966.

Zink AJC and Visocekas R, 1997. Datability of sanidine feldspars using the near infrared TL emission. Radiation Measurements 27: 251 261. 